\title{
Stability and Vapor Pressure of Aqueous Aggregates and Aerosols Containing a Monovalent lon
}

\author{
Yamila A. Perez Sirkin, ${ }^{\dagger}$ Matías H. Factorovich, ${ }^{\dagger}$ Valeria Molinero, $^{\ddagger}{ }^{\circledR}$ and Damián A. Scherlis ${ }^{*},^{\dagger}(0)$ \\ ${ }^{\dagger}$ Departamento de Química Inorgánica, Analítica y Química Física/INQUIMAE, Facultad de Ciencias Exactas y Naturales, \\ Universidad de Buenos Aires, Ciudad Universitaria, Pab. II, Buenos Aires C1428EHA, Argentina \\ ${ }^{\ddagger}$ Department of Chemistry, The University of Utah, 315 South 1400 East, Salt Lake City, Utah 84112-0850, United States
}

ABSTRACT: The incidence of charged particles on the nucleation and the stability of aqueous aggregates and aerosols was reported more than a century ago. Many studies have been conducted ever since to characterize the stability, structure, and nucleation barrier of ion-water droplets. Most of these studies have focused on the free-energy surface as a function of cluster size, with an emphasis on the role of ionic charge and radius. This knowledge is fundamental to go beyond the rudimentary ion-induced classical nucleation theory. In the present article, we address this problem from a different perspective, by computing the vapor pressures of $\left(\mathrm{H}_{2} \mathrm{O}\right)_{n} \mathrm{Li}^{+}$and $\left(\mathrm{H}_{2} \mathrm{O}\right)_{n} \mathrm{Cl}^{-}$aggregates using molecular simulations. Our calculations shed light on the structure, the critical size, the range of stability, and the role of ion-water interactions in aqueous clusters. Moreover, they allow one to assess the accuracy of the classical thermodynamic model, highlighting its strengths and weaknesses.

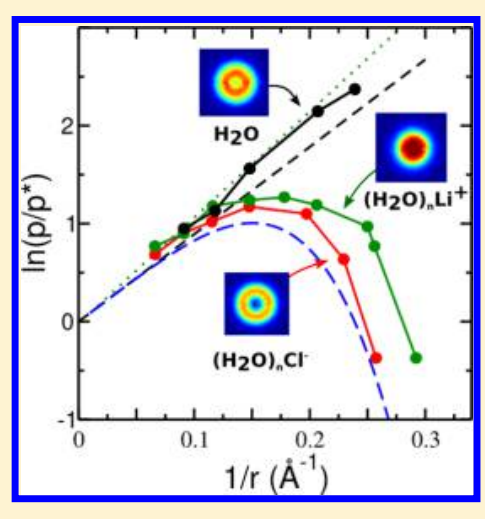

\section{INTRODUCTION}

In a physical chemistry context, the term nucleation alludes to the growth of a new phase from an old one. Nucleation of a liquid from the vapor, or of a solid from the liquid, is accompanied by a decrease in both the enthalpy and the entropy of the nucleating system (whereas the opposite is true for the reverse processes). For a certain chemical potential at which the new phase should be stable, there is a free-energy barrier that needs to be overcome to reach the final state. This barrier depends on a surface tension contribution that predominates for small nuclei but becomes negligible for systems above a critical size. These processes are often characterized through the free-energy curves as a function of nucleus size. ${ }^{1}$

Nucleation of solids or liquids from a vapor phase may proceed either heterogeneously (assisted by a preexisting nucleus or phase) or homogeneously (in the absence of such an assistance). Homogeneous nucleation is a highly activated phenomenon. In the atmosphere, where this process is of high relevance for aerosol formation and growth, nucleation is always heterogeneous. The role of the particles present during the nucleation from the vapor was first pinpointed in 1881 by Aitken, ${ }^{2}$ through the study of mist formation. A few years later, Wilson showed that nucleation rates depended on the nature of these particles. ${ }^{3}$ Nowadays, it is known that the mechanisms of aerosol formation and growth rely on two, three, or possibly more mutually interacting agents, such as sulfur dioxide, volatile organic compounds (VOCs), atmospheric ions, ammonia, amines, organic acids, iodine oxides, and so forth (see ref 4 and citations therein).

Classical nucleation theory $(\mathrm{CNT})^{1,5}$ describes the change in Gibbs free energy $\Delta G$ associated with the transfer of $n$ molecules from the vapor phase to a cluster of radius $r$, according to

$$
\Delta G=-n k T \ln \frac{P}{P^{*}}+4 \pi r^{2} \gamma
$$

where $P$ and $P^{*}$ are the vapor pressures of the aggregate and of the bulk fluid at the same temperature $T, \gamma$ is the surface tension, and $k$ is the Boltzmann constant. ${ }^{4}$ The so-called ion-induced nucleation (IIN) theory takes into account the effect of an anion or cation of charge $q$ by including the interaction of a charged particle of radius $r_{0}$ with a dielectric medium contained in a sphere of radius $r$

$$
\Delta G=-n K T \ln \frac{P}{P^{*}}+4 \pi r^{2} \gamma+\frac{q^{2} e_{0}^{2}}{8 \pi \epsilon_{0}}\left(1-\frac{1}{\epsilon}\right)\left(\frac{1}{r}-\frac{1}{r_{0}}\right)
$$

where $\epsilon$ and $\epsilon_{0}$ are the relative permittivities of the bulk and vacuum phases, respectively. ${ }^{6} \mathrm{~A}$ few modifications to classical IIN theory have been proposed to take into consideration the nature of the ions. ${ }^{7-9}$ The role played by the specific properties of a given ion has been underscored by several simulation studies performed to understand the IIN process. These works have assessed the importance of ionic sign, ${ }^{10,11}$ size, ${ }^{12}$ and polarizability $^{13}$ to conclude that all of these factors, neglected in the IIN nucleation model, must be considered to some extent in order to get a quantitative description of this phenomenon.

In this work, we address the problem of aqueous clusters and aerosols containing monovalent ions from a different perspective, by computing their vapor pressures as a function of size using computer simulations. To this end, we apply the grand

Received: January 19, 2017

Revised: March 9, 2017

Published: March 10, 2017 
canonical screening (GCS) approach, recently developed to compute vapor pressures at interfaces of any kind or shape. ${ }^{14}$ This method has been exhaustively validated against Gibbs Ensemble Monte Carlo and has proved accurate in the calculation of vapor pressures of pure liquids, ${ }^{14}$ aggregates, ${ }^{14,15}$ and solutions. ${ }^{16}$ Using this strategy, in the present article, we establish the range of stability of the aggregates, their critical size, their structure, and the ability of classical thermodynamic theories to describe them.

\section{METHODOLOGY}

Water is described with the rigid SPC/E model, ${ }^{17}$ whereas the ions are represented as point charges with Lennard-Jones potentials, ${ }^{18}$ with $\sigma$ and $\epsilon$ parameters of $2.337 \AA$ and $0.67 \mathrm{~kJ} / \mathrm{mol}$ for $\mathrm{Li}^{+}$and $3.785 \AA$ and $0.52 \mathrm{~kJ} / \mathrm{mol}$ for $\mathrm{Cl}^{-}$. Additionally, we have performed simulations with the $\mathrm{mW}$ coarse-grained model, which represents the water molecule as a single particle interacting through anisotropic short-range forces. ${ }^{19} \mathrm{The}^{+}{ }^{+}$ and $\mathrm{Cl}^{-}$ions are represented within the $\mathrm{mW}$-S force field as the same particle $S$ and without introducing electrostatic interactions. $^{20}$ This model quantitatively reproduces the behavior of $\mathrm{LiCl}$ ions in their effect on water structure, melting temperature of ice, vapor pressure of the solution, and a crossover between crystallization and vitrification as a function of salt concentration. ${ }^{16,20-22}$

The vapor pressure of the ion-containing droplets is computed with the GCS approach, ${ }^{14}$ recently applied to study pure water nanodroplets ${ }^{15}$ and aqueous solutions. ${ }^{16}$ To calculate the vapor pressure of a droplet using this method, independent grand canonical (GC) simulations are performed at different chemical potentials, $\mu$. In each one of these runs, the number of particles may rise or drop, depending on whether the magnitude of $\mu$ is, respectively, above or below the equilibrium value $\mu_{\text {eq }}$. If $\mu>\mu_{\text {eq }}$ condensation occurs leading to a raise of the droplet radius, which in turn, because of the reduction of surface curvature, diminishes the magnitude of $\mu_{\text {eq }}$. In this way, $\mu_{\text {eq }}$ moves gradually away from $\mu$, and the droplet continues to grow until the simulation box is completely filled. Conversely, if $\mu<\mu_{\mathrm{eq}}$ evaporation takes place and the equilibrium pressure increases with surface curvature, accelerating the process to the final state. For pure droplets, the evaporation proceeds until all particles have disappeared. For binary systems, the final state depends on the interaction between the particles; in some cases, a stable configuration corresponding to a minimum in the free-energy surface may be reached that prevents complete loss of solvent molecules. With the procedure described so far, an upper and a lower bound can be established for the equilibrium chemical potential $\mu_{\mathrm{eq}}$ corresponding to the critical size, which is directly connected to the relative vapor pressure if ideality of the gas is assumed. Additionally, in the case of ion-containing clusters, this method provides the size of the free-energy minimum structure for a given $\mu$, if such a minimum exists.

Molecular dynamics simulations were performed using the LAMMPS program ${ }^{23,24}$ for the calculation of droplet radii and the densities presented in Figure 4. Whereas this information could be also extracted from the GC computations described below, the analysis is simpler for a constant number of particles. The equations of motion were integrated using the Verlet algorithm with a time step of $1 \mathrm{fs}(\mathrm{SPC} / \mathrm{E})$ or $5 \mathrm{fs}(\mathrm{mW})$. The temperature was controlled with the Nosé-Hoover thermostat at $298 \mathrm{~K}$ with a relaxation time of $0.25 \mathrm{ps}$.

For the calculation of vapor pressures, grand canonical Monte Carlo (GCMC) simulations at $298 \mathrm{~K}$ were carried out with version 7.1.0 of the MCCCS Towhee code. ${ }^{25,26}$ The calculations were performed in periodic boundary conditions in a box of $60 \times$ $60 \times 60 \AA^{3}$, using a long-range cutoff of $15 \AA$ for the Coulomb contribution, which is enough to include all interactions between the molecules in the largest aggregate, excluding at the same time the interactions between periodic images. Three kinds of moves are possible with equal probability: rotation, displacement, or insertion/deletion. If the third kind turns out, then the algorithm proceeds to randomly choose between particle deletion or insertion with equal probability. The magnitudes of displacements and rotations were adjusted on the fly to fit a $50 \%$ acceptance ratio. The acceptance criterion for the exchange of water molecules is based on the chemical potential $\mu$, which is related to the vapor pressure by $\mu=\mu^{\theta}(T)+k_{\mathrm{B}} T \ln \frac{P \phi(P)}{P^{\theta}}$, where $\phi$ is the fugacity coefficient. In the Towhee code, the total, absolute chemical potential of a component $i$ is defined and computed as follows ${ }^{26}$

$$
\mu_{\text {total }}(i)=k_{\mathrm{B}} T \ln \left(\left\langle\frac{W \cdot V}{\left.(N(i)+1) \cdot \Lambda^{3}(i)\right)}\right\rangle\right)
$$

where $W$ is the difference in total energy after inserting a test particle $i$ on the system, $V$ is the volume, $N(i)$ is the number of particles, and $\Lambda(i)$ is the thermal de Broglie wavelength. The brackets, \langle\rangle , denote an average on the insertion moves. This "methodological" definition of the chemical potential allows its calculation through an insertion method-the Towhee code employs Rosenbluths weights. This definition of chemical potential leaves the reference state $\mu^{\theta}$ as an unknown constant, and therefore, the pressure is not directly available. Nevertheless, Towhee can compute the chemical potential $\mu_{\text {total }}(i)^{N P T}$ (or simply $\mu^{N P T}$ ) through the formula above in an additional Monte Carlo simulation in the isothermal-isobaric (NPT) ensemble performed at a known $P^{N P T}$. Writing down the chemical potentials in both ensembles, we have

$$
\begin{aligned}
\mu^{N P T} & =\mu^{\theta}(T)+k_{\mathrm{B}} T \ln \frac{P^{N P T} \phi\left(P^{N P T}\right)}{P^{\theta}} \\
\mu^{\mathrm{GCMC}} & =\mu^{\theta}(T)+k_{\mathrm{B}} T \ln \frac{P^{\mathrm{GCMC}} \phi\left(P^{\mathrm{GCMC}}\right)}{P^{\theta}}
\end{aligned}
$$

Combining these two equations, we obtain the following formula for the pressure in the GCMC simulation

$$
P^{\mathrm{GCMC}}=P^{N P T} \exp \left(\frac{\mu^{\mathrm{GCMC}}-\mu^{N P T}}{k_{\mathrm{B}} T}\right) \cdot \frac{\phi\left(P^{N P T}\right)}{\phi\left(P^{\mathrm{GCMC}}\right)}
$$

which can be used to retrieve the pressure $P^{\mathrm{GCMC}}$ for any given $\mu^{\mathrm{GCMC}}$. Assuming the ideal gas approximation, or if $P^{\mathrm{GCMC}}$ and $P^{N P T}$ are very close, the ratio between the fugacity coefficients can be taken to be 1 in eq 3 . In particular, the ideal gas approximation is frequently adopted in GC simulations of $\mathrm{H}_{2} \mathrm{O}$ near ambient or evaporation conditions. ${ }^{14,27-30}$

\section{RESULTS AND DISCUSSION}

Figure 1 presents the logarithm of the saturation ratio $S$ as a function of the inverse radius, for aqueous clusters at $298 \mathrm{~K}$ ( $S=$ $P / P^{*}$, with $P$ and $P^{*}$ the vapor pressure of the aggregate and that of bulk water, respectively). The solid curves show the results obtained with the SPC/E model and the GCS approach for pure water clusters (black) and for aqueous clusters containing a single $\mathrm{Cl}^{-}$(green) or $\mathrm{Li}^{+}$(red) ion. The black dashed line 


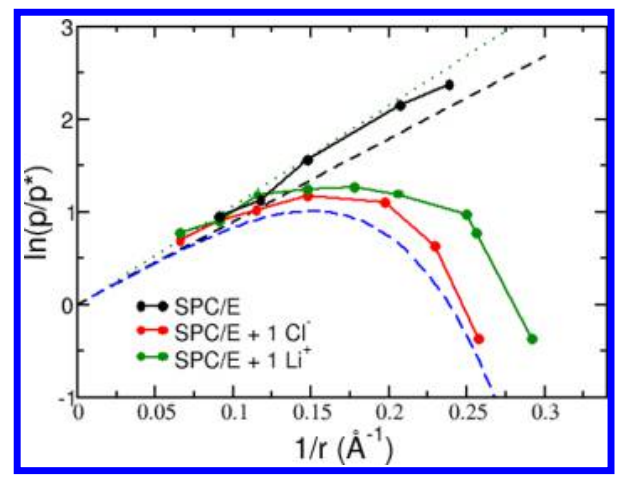

Figure 1. Logarithm of the relative vapor pressure as a function of the inverse radius of the droplets. Black, red, and green lines represent results obtained with the SPC/E model for pure water, water $-\mathrm{Cl}^{-}$, and water $-\mathrm{Li}^{+}$aggregates, respectively. The black dashed and dotted lines show the predictions of the Kelvin equation, with and without the Tolman correction.

corresponds to the relative pressure predicted by the Kelvin equation for water droplets

$$
\ln S=\ln \frac{P}{P^{*}}=\frac{2 \gamma V_{\mathrm{m}}}{r R T}
$$

where $\gamma$ is the surface tension of the bulk liquid, $V_{\mathrm{m}}$ is the molar volume, $R$ is the ideal gas constant, and $r$ is the radius from the center of mass to the equimolar surface (for details on the calculation of the equimolar radius, see ref 14). It can be seen that all curves obey the Kelvin equation for radii above $\sim 8 \AA(0.125$ $\AA^{-1}$ ), or only $\sim 80 \mathrm{H}_{2} \mathrm{O}$ molecules. In the case of pure water, the Kelvin equation with the Tolman correction ${ }^{31}$

$$
\ln \frac{P}{P^{*}}=\frac{2 \gamma V_{\mathrm{m}}}{r R T} \cdot \frac{r-\delta}{r+\delta}
$$

provides a good description of the vapor pressure up to a radius as small as $5 \AA$, below which a discrepancy starts to be manifest. The observed trend corresponds to a negative Tolman length, $\delta$ $\approx-0.3 \AA$. This negative deviation implies that the surface tension increases as the droplet becomes nanoscopic, in qualitative agreement with previous estimates for the SPC/E model. ${ }^{32}$ The validity of classical thermodynamics for pure water droplets at such small length scales has been analyzed by us in a recent study using the $\mathrm{mW}$ coarse-grained potential. ${ }^{15}$ The present results obtained with the atomistic SPC/E model also show the applicability of the Kelvin equation down to the same range.

The behavior of the clusters containing a single ion is qualitatively different; the curves show a maximum at $S \approx 3-3.5$ and $n \approx 30$, indicating that above this pressure the clusters will not be stable against the bulk phase. At a given pressure or chemical potential lying below this maximum (and above $P^{*}$ ), the aggregate will present two stable or metastable forms; the left-hand branch, at large radii $\left(1 / r<0.15 \AA^{-1}\right)$, corresponds to maxima in the free-energy surface, that is, metastable points with respect to the chemical potential, which reflect the critical cluster size. The right section of the curve, on the other hand, represents free-energy minima. These two extremes can be appreciated in Figure 2, which is a depiction of the free energy as a function of size for the $\left(\mathrm{H}_{2} \mathrm{O}\right)_{n} \mathrm{Li}^{+}$cluster at two different saturation ratios. This figure portrays the number of molecules at the minima and maxima on the free-energy surface, as resulting from the GCS approach.

For $n>80$, the left-hand branch of the curves for the $\mathrm{Li}^{+}$and $\mathrm{Cl}^{-}$clusters in Figure 1 essentially reproduces the behavior of

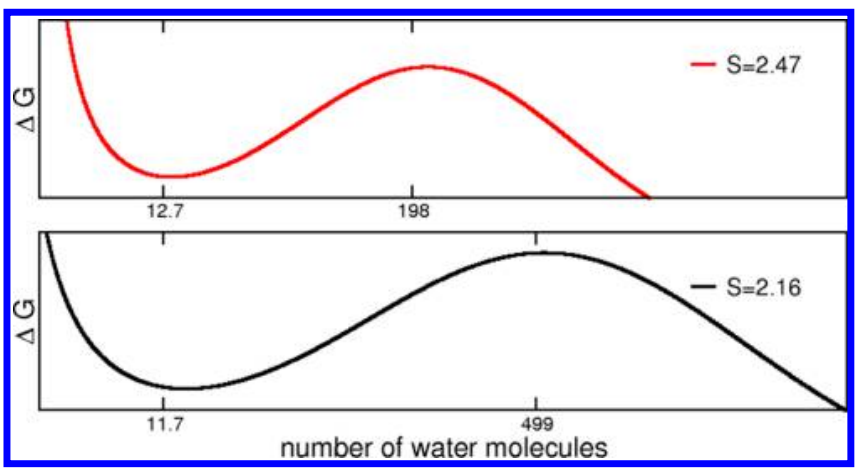

Figure 2. Schematic representation of the excess Gibbs energy as a function of the number of water molecules for $\left(\mathrm{H}_{2} \mathrm{O}\right)_{n} \mathrm{Li}^{+}$aggregates at two different saturation ratios.

pure water droplets. In other words, when the size of the aggregate is above 80 molecules $(r>8 \AA)$, the effect of the ion on the vapor pressure, and presumably on the free energy, becomes negligible. On the other hand, it can be seen that the critical size is extremely sensitive to the chemical potential. Figure 2 highlights that a change of just $15 \%$ in the pressure more than doubles the critical cluster size, from 198 to 499 molecules.

The evaporation of water clusters, or clusters of any other pure liquid, can be regarded as an autocatalytic process; as they lose mass and shrink, the increase in surface curvature raises their vapor pressure, which accelerates the whole sequence downhill in the free-energy landscape toward complete disintegration. In the presence of an ion, however, the evaporation process may get trapped in a minimum consisting of a few solvent molecules surrounding the charged particle. Our GC simulations spontaneously lead to these minima if the starting configuration lies to the left of the critical cluster size. The number of water molecules forming these free-energy minimum structures depends on the chemical potential; for $\mathrm{Li}^{+}$, it varies from 9 at $S$ $=0.7$ to 13 at $S=2.5$. For $\mathrm{Cl}^{-}$, these numbers are slightly smaller at low pressures $(n \approx 7$ for $S=0.7)$ but may be larger than those for $\mathrm{Li}^{+}$clusters at higher saturation ratios. These values are very close to those found by Bin Chen and coauthors with the TIP4P model for ions of 3 and $4 \AA$ diameter. ${ }^{13}$

The stability of these binary aggregates results from the balance between two opposing driving forces: on one hand, the hydration energy of the electrolyte, and on the other, the surface curvature. A rising saturation ratio tends to reduce the critical radius because higher curvature raises the vapor pressure and at the same time promotes an increase in the size of the minimum, driven by the hydration free energy (the sensitivity of this size with respect to pressure is relatively minor, as reflected in the slope of the right-hand branch in Figure 1). Because of these opposite trends, as the pressure goes up, the minimum and the maximum approach each other to eventually merge at $S \approx 3$. Above this saturation ratio, the free-energy curve turns out to be monotonically decreasing, that is, clusters containing a $\mathrm{Li}^{+}$or $\mathrm{Cl}^{-}$ ion become unstable with respect to the solution.

In the framework of IIN theory, it is assumed that the radius of the droplet is proportional to $n^{1 / 3}\left(V=n / \rho=\frac{4}{3} \pi r^{3}\right)$. Figure 3 shows the dependence of the equimolar radius on the number of molecules, indicating that this assumption is tightly observed for all of the aqueous clusters examined in this work down to $n \approx 50$. Interestingly, this linear relation remains valid for clusters of only 20 molecules in the case of pure water and even less in that of the water $-\mathrm{Li}^{+}$aggregate. For $\mathrm{Cl}^{-}$, the radius exhibits a positive 


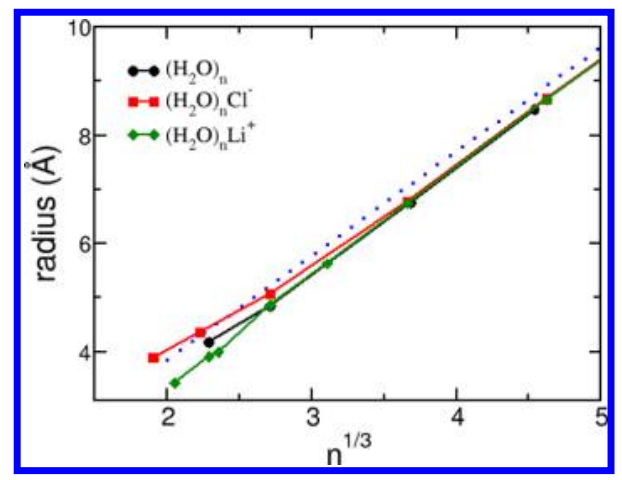

Figure 3. Equimolar radius of the cluster as a function of the cube root of the number of water molecules, $n$. The dotted line represents the linear relationship $r=\left(\frac{3}{4 \pi \rho}\right)^{1 / 3} n^{1 / 3}$, with $\rho=0.0334 \AA^{-3}$.

deviation when $n<50$. This deviation originates from the hydration structure of the anion, which involves a shell of water molecules with the hydrogen atoms facing the negative charge, leaving an excluded volume that results in a larger radius for the same number of molecules, in comparison with pure water or water- $\mathrm{Li}^{+}$clusters. This effect is clearly visible in the contour maps collected in Figure 4, showing for all three systems the time-averaged density projected on $2 \mathrm{D}$ for $n=12$. It can be seen that also in the case of pure water the hydrogen bond network favors a hollow region in the central part of the cluster. In recent work, ${ }^{15}$ we have identified this particular feature in $\left(\mathrm{H}_{2} \mathrm{O}\right)_{9}$ aggregates using both the $\mathrm{mW}$ model and first-principles DFT simulations. This arrangement seems exacerbated in the presence of $\mathrm{Cl}^{-}$, while it is disarticulated by the lithium cation, which facilitates a more compact configuration where the oxygen atoms are oriented toward the charge. Figure 5 displays two typical structures for $\mathrm{Li}$ and $\mathrm{Cl}$ clusters extracted from molecular dynamics trajectories, illustrating the distinctive arrangements of the solvent around the ion in each case. This difference in the radii of the clusters explains, to some extent, the separation of the curves corresponding to chloride and lithium aggregates in the limit of small sizes in Figure 1. In addition, for a given number of $\mathrm{H}_{2} \mathrm{O}$ molecules, the stability of small water $-\mathrm{Cl}^{-}$clusters appears in general marginally higher than that of water- $-\mathrm{Li}^{+}$clusters. This is depicted in Figure 6. The lower vapor pressure of the chloride aggregates is seemingly inconsistent with the higher hydration energy of $\mathrm{Li}^{+}$, around $30-50 \%$ larger than that of $\mathrm{Cl}^{-}$according to experimental estimates. ${ }^{33}$ The explanation is likely to be found in the hydrogen bond network arising in the solvation shell; the

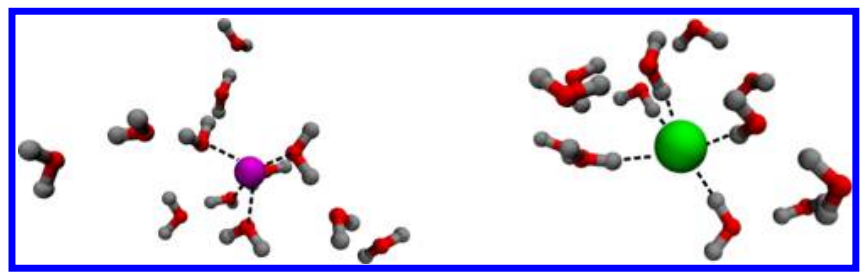

Figure 5. Instantaneous configurations of $\left(\mathrm{H}_{2} \mathrm{O}\right)_{12} \mathrm{Li}^{+}$(left) and $\left(\mathrm{H}_{2} \mathrm{O}\right)_{12} \mathrm{Cl}^{-}$(right) extracted from molecular dynamics simulations at $298 \mathrm{~K}$. The dashed lines depict hydrogen bonds between water and the ion.

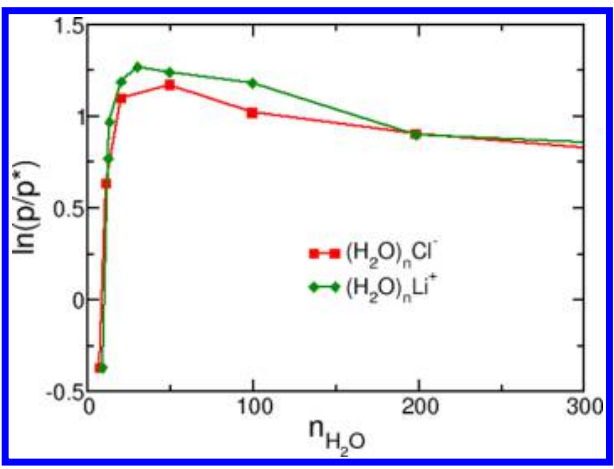

Figure 6. Logarithm of the relative vapor pressure as a function of the number of water molecules for water $-\mathrm{Cl}^{-}$and water $-\mathrm{Li}^{+}$clusters.

strong interaction of water molecules with $\mathrm{Li}^{+}$may restrain the flexibility of the shell, precluding the optimization of the hydrogen bonds and further stabilization of the system.

The IIN considers the interaction of a charged particle with a uniform dielectric medium enclosed in a sphere of radius $r$, neglecting the molecular structure of the solvent and the nature of the ion (eq 2). Derivation of eq 2 with respect to $r$ leads to an expression for the logarithm of $S$ as a function of the droplet radius, known as the Kelvin-Thomson equation

$$
\ln S=\ln \frac{P}{P^{*}}=\frac{2 \gamma V_{\mathrm{m}}}{R T} \frac{1}{r}-\frac{q^{2} e_{0}^{2} V_{\mathrm{m}}}{32 \pi^{2} \epsilon_{0}}\left(1-\frac{1}{\epsilon_{l}}\right) \frac{1}{r^{4}}
$$

This formula is plotted in Figure 7, together with the results from the GC simulations. Interestingly, the IIN model gives a fair account of the saturation ratio up to $r \approx 7 \AA$, beyond which it starts to fail. For $\left(\mathrm{H}_{2} \mathrm{O}\right)_{n} \mathrm{Cl}^{-}$clusters, the discrepancies are on the order of 15-25\%; however, for the lithium-water clusters, the deviation becomes significant, especially in the limit of small

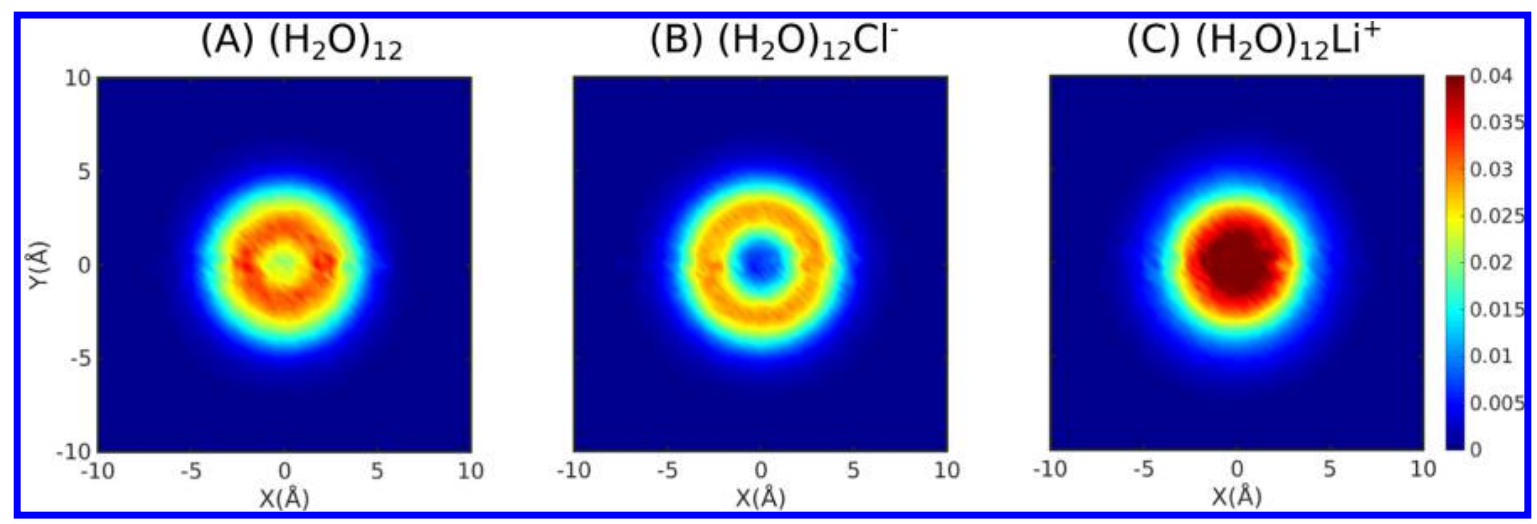

Figure 4. Two-dimensional contour maps showing the density of oxygen atoms, for aqueous aggregates at $298 \mathrm{~K}$. Units for the color scale bar are $\AA^{-3}$. The densities were averaged over time windows of $25 \mathrm{~ns}$. In $\left(\mathrm{H}_{2} \mathrm{O}\right)_{12} \mathrm{Li}^{+}$and $\left(\mathrm{H}_{2} \mathrm{O}\right)_{12} \mathrm{Cl}^{-}$, the ions reside on average in the center of mass. 


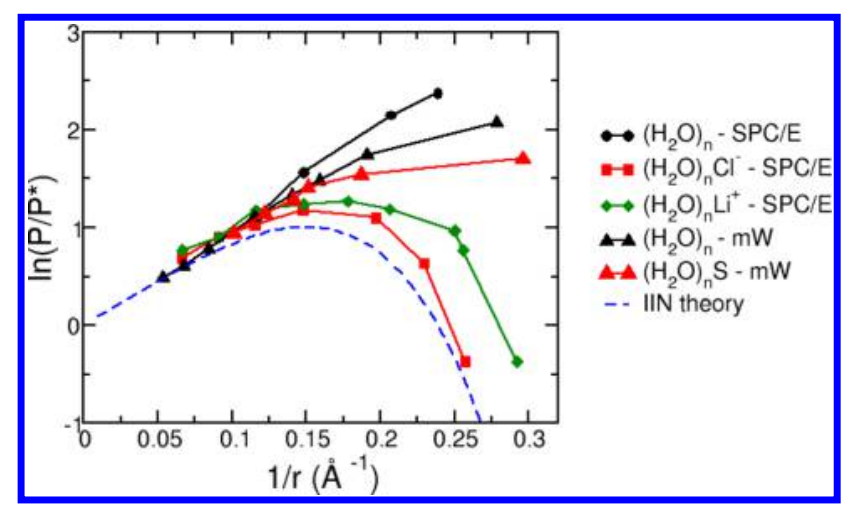

Figure 7. Logarithm of the relative vapor pressure as a function of the inverse radius of the droplets. Legends are the same as those in Figure 1. Here, the triangles identify the results obtained with the $\mathrm{mW}$ coarsegrained model, for clusters of pure water (black) and with a single ion (red). The blue dashed line shows the prediction of classical IIN theory.

droplets, where the theory underestimates the vapor pressure by a factor of 3 or 4 . Assumptions of the IIN, for example, that the cluster is a continuous medium of homogeneous density and permittivity with an ion at the center, start to be too crude for clusters of just 30 or 20 molecules. The more acceptable agreement obtained for the vapor pressure of chloride-water clusters is likely to be related to a smaller interaction energy; the IIN model, which represents the system as a charged particle embedded in a homogeneous dielectric, will fail more dramatically for the case of large hydration energies capable of exacerbating the structure of the solvation shell and inducing strong specific interactions with the solvent.

Recently, we successfully employed the $\mathrm{mW}$ coarse-grained model to investigate the vapor pressure of lithium and sodium chloride solutions. ${ }^{16}$ This model has shown that, despite the lack of electrostatics, it could provide a good description of the activity coefficients and therefore of the relative vapor pressure of these electrolyte solutions, thanks to a parametrization strategy based on reproducing some fundamental features of the solution, such as solvation structure and effective association between ions. The success of the models appears at odds with a severely underestimated hydration free energy $(-22.2 \mathrm{kcal} / \mathrm{mol}$ for the $S$ particle against $\sim-120$ and $\sim-80 \mathrm{kcal} / \mathrm{mol}$ for experimental $\mathrm{Li}^{+}$ and $\mathrm{Cl}^{-}$, respectively), which turns out to be compensated by the underestimation of the vaporization free energy of the salt. ${ }^{16}$ In the present case, however, the activity coefficient of the bulk does not reflect the behavior in the aggregate, and a too weak hydration free energy leads to too high vapor pressures. This is depicted in Figure 7, where it is shown that the saturation ratios of the $\mathrm{mW}$ ion-containing aggregates hardly deviate from those of pure water clusters. As a matter of fact, the coarse-grained $\mathrm{mW}$-S model is unable to produce stable aggregates. Figure 8 presents the number of water molecules as a function of the Monte Carlo step for GC simulations based on the atomistic $\mathrm{SPC} / \mathrm{E}$ and coarse-grained $\mathrm{mW}$-S force fields. The figure shows for every model the results of two simulations, one above and one below the equilibrium chemical potential. Whereas in the case of the SPC/E model, the system reaches a stable, free-energy minimum structure, for the $\mathrm{mW}$ simulations, the number of particles drops to zero, leaving the bare ion in the simulation box.

\section{CONCLUDING REMARKS}

The present atomistic SPC/E simulations indicate that the Kelvin equation can be applied to describe the saturation ratio of

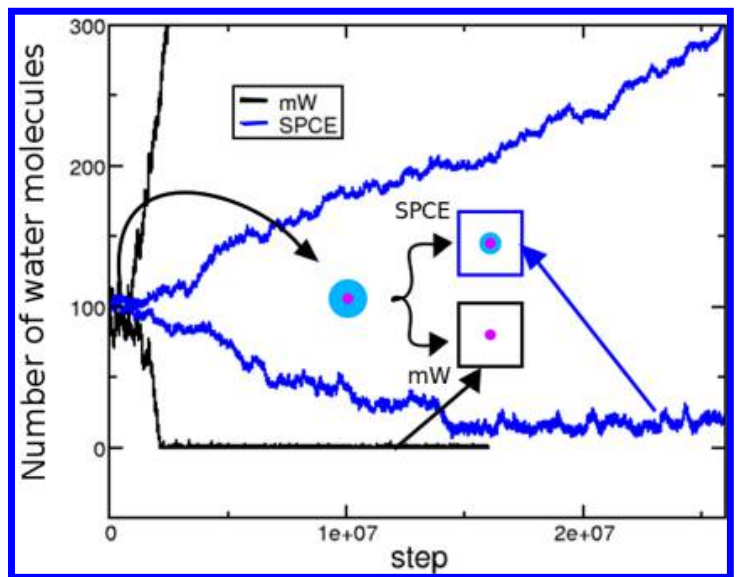

Figure 8. Number of water molecules as a function of the Monte Carlo step in SPC/E and $\mathrm{mW} \mathrm{GC} \mathrm{simulations} \mathrm{of} \mathrm{clusters} \mathrm{containing} \mathrm{an} \mathrm{ion.}$ The curves with positive slope correspond to a chemical potential above the equilibrium value, and vice versa. The $\mathrm{mW}$ model does not produce a stable aggregate but evaporates completely. For the atomistic model, the curves for evaporation and condensation correspond to $S=2.90$ and 3.69 , respectively. For the coarse-grained simulations, these values are 2.90 and 2.98

water clusters as small as $1 \mathrm{~nm}$ diameter, in agreement with previous coarse-grained results. ${ }^{15}$ Moreover, our computations suggest that curvature in small SPC/E aggregates produces an increase in the surface tension, consistent with the data reported by Sedlmeier and Netz. ${ }^{32}$

Clusters containing a single $\mathrm{Li}^{+}$or $\mathrm{Cl}^{-}$ion may be stable or metastable up to saturation ratios of $\sim 3-3.5$, above which they become unstable against the bulk phase. The presence of monovalent ions does not seem to significantly affect the thermodynamics of clusters exceeding 80 water molecules. These coexist with smaller, stable structures containing typically less than 20 water molecules, which constitute minima in the freeenergy surface. We have characterized these structures as a function of pressure to find that the number of water molecules in lithium and chloride clusters is about the same, going from $\sim 13$ molecules at $S=2.5$ to $\sim 8$ at $S=0.7$. In the limit of small aggregates, $\left(\mathrm{H}_{2} \mathrm{O}\right)_{n} \mathrm{Cl}^{-}$systems can be significantly larger than $\left(\mathrm{H}_{2} \mathrm{O}\right)_{n} \mathrm{Li}^{+}$systems due to the organization of water molecules around the central ion. This difference tends to disappear altogether for $n>50$. Within this range, the vapor pressure of lithium aggregates is slightly larger, which may be explained in terms of a suboptimal hydrogen bond arrangement of water around the $\mathrm{Li}^{+}$ion. The higher hydration energy of the cation in comparison with that of $\mathrm{Cl}^{-}$must induce a more compact and rigid solvation shell with a lower stability. Thus, the vapor pressure results from a delicate balance between the interaction of the $\mathrm{H}_{2} \mathrm{O}$ molecules with themselves and with the ion.

Despite its crude assumptions, the classical IIN theory provides a reasonable guess of the radius corresponding to the maximum supersaturation attainable. However, it tends to underestimate the vapor pressure of the clusters. This underestimation does not seem so dramatic for moderate hydration energies but may be withering for small droplets and high ionwater interactions, reaching in some cases a factor of 3 or more. This highlights the importance of refined models or molecular simulations in describing the thermodynamics and nucleation of aerosols in the presence of ions. 


\section{AUTHOR INFORMATION}

\section{Corresponding Author}

*E-mail: damian@qi.fcen.uba.ar. Phone: 54-11-45763378 ext 123.

\section{ORCID}

Valeria Molinero: 0000-0002-8577-4675

Damián A. Scherlis: 0000-0002-0588-287X

\section{Notes}

The authors declare no competing financial interest.

\section{ACKNOWLEDGMENTS}

This study has been supported by a collaborative grant of the Agencia Nacional de Promoción Científica y Tecnológica de Argentina (PICT 2012-2292 to V.M. and D.A.S.) and by The University of Buenos Aires, UBACYT 20020120100333BA. We thank the Center of High Performance Computing of the University of Utah for an award of computing time and technical support.

\section{REFERENCES}

(1) Zhang, R. Getting to the Critical Nucleus of Aerosol Formation. Science 2010, 328, 1366-1367.

(2) Aitken, J., XII On Dust, Fogs, and Clouds. Trans. - R. Soc. Edinburgh 1881, 30, 337-368

(3) Wilson, C. T. R. Condensation of Water Vapour in the Presence of Dust-Free Air and other Gases. Proc. R. Soc. London 1897, 61, 240-242.

(4) Zhang, R.; Khalizov, A.; Wang, L.; Hu, M.; Xu, W. Nucleation and Growth of Nanoparticles in the Atmosphere. Chem. Rev. 2012, 112, 1957-2011.

(5) Rodebush, W. The Vapor Pressure of Small Drops. Proc. Natl. Acad. Sci. U. S. A. 1954, 40, 789-794.

(6) Thomson, J. J. Applications of Dynamics to Physics and Chemistry; Macmillan, 1888.

(7) Shchekin, A. K.; Podguzova, T. S. The Modified Thomson Equation in the Theory of Heterogeneous Vapor Nucleation on Charged Solid Particles. Atmos. Res. 2011, 101, 493-502.

(8) Chan, L. Y.; Mohnen, V. Ion Nucleation Theory. I. Atmos. Sci. 1980, 37, 2323-2331.

(9) Nadykto, A. B.; Yu, F. Dipole Moment of Condensing Monomers: A New Parameter Controlling the Ion-Induced Nucleation. Phvs. Rev. Lett. 2004, 93, 016101.

(10) Oh, K.; Gao, G.; Zeng, X. C. Nucleation of Water and Methanol Droplets on Cations and Anions: the Sign Preference. Phvs. Rev. Lett. 2001, 86, 5080.

(11) Nadykto, A. B.; Al Natsheh, A.; Yu, F.; Mikkelsen, K.; Ruuskanen, J. Quantum Nature of the Sign Preference in Ion-Induced Nucleation. Phvs. Rev. Lett. 2006, 96, 125701.

(12) Kathmann, S. M.; Schenter, G. K.; Garrett, B. C. Ion-Induced Nucleation: The Importance of Chemistry. Phys. Rev. Lett. 2005, 94, 116104.

(13) Keasler, S. J.; Kim, H.; Chen, B. Ion-Induced Nucleation: The Importance of Ionic Polarizability. I. Phys. Chem. A 2010, 114, 45954600.

(14) Factorovich, M. H.; Molinero, V.; Scherlis, D. A. A simple Grand Canonical Approach to Compute the Vapor Pressure of Bulk and Finite Size Systems. I. Chem. Phys. 2014, 140, 064111.

(15) Factorovich, M. H.; Molinero, V.; Scherlis, D. A. Vapor Pressure of Water Nanodroplets. I. Am. Chem. Soc. 2014, 136, 4508-4514.

(16) Perez Sirkin, Y. A.; Factorovich, M. H.; Molinero, V.; Scherlis, D. A. Vapor pressure of Aqueous Solutions of Electrolytes Reproduced with Coarse-Grained Models Without Electrostatics. I. Chem. Theorv Comput. 2016, 12, 2942-2949.

(17) Berendsen, H.; Grigera, J.; Straatsma, T. The Missing Term in Effective Pair Potentials. I. Phys. Chem. 1987, 91, 6269-6271.
(18) Lee, S. H.; Rasaiah, J. C. Molecular Dynamics Simulation of Ion Mobility. 2. Alkali Metal and Halide Ions Using the SPC/E Model for Water at 25 C. I. Phvs. Chem. 1996, 100, 1420-1425.

(19) Molinero, V.; Moore, E. B. Water Modeled As an Intermediate Element between Carbon and Silicon. I.Phvs. Chem. B 2009, 113, 40084016.

(20) Le, L.; Molinero, V. Nanophase Segregation in Supercooled Aqueous Solutions and Their Glasses Driven by the Polyamorphism of Water. I. Phys. Chem. A 2011, 115, 5900-5907.

(21) Bullock, G.; Molinero, V. Low-Density Liquid Water is the Mother of Ice: on the Relation between Mesostructure, Thermodynamics and Ice Crystallization in Solutions. Faradav Discuss. 2014, 167, $371-388$.

(22) Hudait, A.; Molinero, V. Ice Crystallization in Ultrafine WaterSalt Aerosols: Nucleation, Ice-Solution Equilibrium, and Internal Structure. I. Am. Chem. Soc. 2014, 136, 8081-8093.

(23) Plimpton, S. Fast Parallel Algorithms for Short-Range Molecular Dynamics. I. Comput. Phvs. 1995, 117, 1-19.

(24) LAMMPS web page. http://lammps.sandia.gov/ (accessed March $8,2017)$.

(25) Martin, M. G. MCCCS Towhee: A Tool for Monte Carlo Molecular Simulation. Mol. Simul. 2013, 39, 1212-1222.

(26) MCCCS Towhee web page. http://towhee.sourceforge.net (accessed March 8, 2017).

(27) Puibasset, J.; Pellenq, R. J.-M. Water Adsorption on Hydrophilic Mesoporous and Plane Silica Substrates: A grand canonical Monte Carlo Simulation Study. I. Chem. Phvs. 2003, 118, 5613-5622.

(28) Puibasset, J.; Pellenq, R. J.-M. Grand Canonical Monte Carlo Simulation Study of Water Adsorption in Silicalite at 300 K. I. Phvs. Chem. B 2008, 112, 6390-6397.

(29) Malani, A.; Ayappa, K. G. Adsorption Isotherms of Water on Mica: Redistribution and Film Growth. I. Phvs. Chem. B 2009, 113, $1058-1067$.

(30) Tombácz, E.; Hajdú, A.; Illés, E.; László, K.; Garberoglio, G.; Jedlovszky, P. Water in Contact with Magnetite Nanoparticles, as Seen from Experiments and Computer Simulations. Langmuir 2009, 25, 13007-13014.

(31) Tolman, R. C. The Effect of Droplet Size on Surface Tension. $L$. Chem. Phys. 1949, 17, 333-337.

(32) Sedlmeier, F.; Netz, R. R. The Spontaneous Curvature of the Water-Hydrophobe Interface. I. Chem. Phvs. 2012, 137, 135102.

(33) Lamoureux, G.; Roux, B. Absolute Hydration Free Energy Scale for Alkali and Halide Ions Established from Simulations with a Polarizable Force Field. I. Phvs. Chem. B 2006, 110, 3308-3322. 